\title{
Correction to: Gradient shrinking Ricci solitons of half harmonic Weyl curvature
}

\author{
Jia-Yong $\mathrm{Wu}^{1} \cdot$ Peng $\mathrm{Wu}^{2} \cdot$ William Wylie $^{3}$ \\ Published online: 8 April 2021 \\ ○ Springer-Verlag GmbH Germany, part of Springer Nature 2021
}

\section{Correction to: Calc Var 57:141 (2018) https://doi.org/10.1007/s00526-018-1415-x}

Proposition 3.1 of our manuscript (arXiv:1410.7303) cited Theorem 1.1 of Cao-Tran announced paper in 2013 (arXiv:1311.0846). But our published paper [2] missed the citation [1] due to the corresponding author's negligence, which we did not notice in the final proofs, and we would like to add now with this erratum. In fact, Proposition 3.1 in [2] is the earlier Theorem 1.1 in [1].

\section{References}

1. Cao, X.-D., Tran, H.: The Weyl tensor of gradient Ricci solitons. Geom. Topol. 20, 389-436 (2016)

2. Wu, J.-Y., Wu, P., Wylie, W.: Gradient shrinking Ricci solitons of half harmonic Weyl curvature. Calc. Var. 57, $141(2018)$

Publisher's Note Springer Nature remains neutral with regard to jurisdictional claims in published maps and institutional affiliations.

The original article can be found online at https://doi.org/10.1007/s00526-018-1415-x.

$凶 \quad$ Peng Wu

wupenguin@fudan.edu.cn

Jia-Yong Wu

jywu81@yahoo.com

William Wylie

wwylie@syr.edu

1 Department of Mathematics, Shanghai Maritime University, Haigang Avenue 1550, Shanghai 201306, People's Republic of China

2 Shanghai Center for Mathematical Sciences, Fudan University, Shanghai 200433, People's Republic of China

3 Department of Mathematics, Syracuse University, Syracuse, NY 13244, USA 